Research Paper

\title{
Radical Nephrectomy with or without Lymph Node Dissection for PT3 Renal Cell Carcinoma: A Propensity Score-based Analysis
}

\author{
Pin $\mathrm{Li}^{1}{ }^{2}$, Cheng Peng $23^{*}$, Liangyou Gu${ }^{2}$, Yongpeng Xie ${ }^{4}$, Wenyuan $\mathrm{Nie}^{5}$, Yu Zhang ${ }^{2}$, Yundong Xuan²,

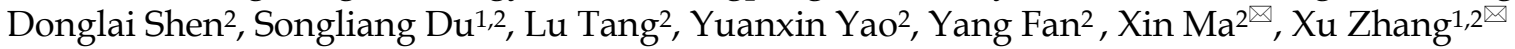 \\ 1. School of Medicine, Nankai University, Tianjin, People's Republic of China \\ 2. Department of Urology, State Key Laboratory of Kidney Diseases, the First Medical Center of Chinese PLA General Hospital, Beijing, People's Republic of \\ China \\ 3. Department of Urology, the Seventh Medical Center of Chinese PLA General Hospital, Beijing, People's Republic of China. \\ 4. Department of Urology, the First Affiliated Hospital of Chongqing Medical University, Chongqing, People's Republic of China \\ 5. Department of Urology, Chinese People's Liberation Army, 89th Hospital, Weifang, Shandong, People's Republic of China. \\ ${ }^{*}$ P.L. and C.P. contributed equally to this research.
}

$\triangle$ Corresponding authors: Xu Zhang, Department of Urology, Chinese PLA General Hospital, Beijing 100853, People's Republic of China, Tel.: +86-10-6693-8211, Fax: +86-10-6822-3567. E-mail addresses: xzhang@foxmail.com or Xin Ma, Department of Urology, Chinese PLA General Hospital, Beijing 100853, People's Republic of China, Tel.: +86-10-6693-8008, Fax: +86-10-6822-3575. E-mail addresses: urologist@foxmail.com

(c) Ivyspring International Publisher. This is an open access article distributed under the terms of the Creative Commons Attribution (CC BY-NC) license (https://creativecommons.org/licenses/by-nc/4.0/). See http://ivyspring.com/terms for full terms and conditions.

Received: 2018.10.02; Accepted: 2019.04.25; Published: 2019.05.26

\begin{abstract}
Objectives: To study whether radical nephrectomy (RN) with lymph node dissection (LND) can benefit PT3 renal cell carcinoma (RCC) patients versus no LND under the 2018 American Joint Committee on Cancer TNM classification system.

Subjects/Patients and Methods: We performed a retrospective cohort study of clinicopathological data for 245 T3 RCC patients, who underwent radical nephrectomy between January 2006 and December 2013 at our center, including 67 (27.1\%) who underwent LND. The relationships between the LND and progression-free survival (PFS), cancer-specific survival (CSS), and overall survival (OS) were evaluated using 1:1 propensity score (PS) matching. Then, Kaplan-Meier survival analysis and Cox regression analysis were conducted to study whether these patients can benefit from LND. Depending on the LND number, we divided the cohort into two groups for further comparation. At last, we validated the results with the TCGA database KIRC patients.

Results: The median follow-up time was 4.9 years. Sixty-seven pairs of patients were screened by the PS and were further analyzed. We conducted a Cox regression with the survival data and found that the LND group, compared with the non-LND group, showed no survival benefit on PFS, CSS, and OS ( $p=0.444,0.809$, and 0.816 , respectively). However, the removal of 5 or more LNs showed negative effect on OS $(P=0.0387)$. TCGA cohort results are mostly consistent with our findings.

Conclusion: RN with LND cannot improve the PFS, CSS, or OS for $\mathrm{PT} 3$ renal cell carcinoma patients.
\end{abstract}

Key words: kidney cancer, renal cell carcinoma, lymph node dissection, propensity score

\section{Introduction}

LND is a general surgery strategy for oncology. However, unlike other urogenital cancers, such as penile, bladder, or prostate cancer, the effect of LND as a alternative treatment for patients with RCC is still controversial. The only piece of evidence regarding the treatment is the prospective, randomizedcontrolled trial EORTC 30881. Owing to the modification of the tumor, node, and metastases 
(TNM) classification system in 1978 and the low synthesize positive lymph node probability, the conclusions have been restricted [1]. Previously, a series of retrospective studies and meta-analyses have indicated that LND is unnecessary, even in high-risk RCC patients [2-4]. Several other retrospective studies have shown that LND improved the survival of RCC patients [5-7]. Nevertheless, in the 2018 EAU guidelines for renal cell carcinoma, LND was also recommended for high-risk renal cell carcinoma patients with evidence of level $2 \mathrm{~b}$ disease [8]. Meanwhile, the American Joint Committee on Cancer (AJCC) updated the standards for the TNM system for RCC and placed invasion of the pelvicalyceal system under T3a. Given that there are so many confounding factors in this field, more accurate patient sampling and reducing relevant variables' influences may bring new insights into this problem. Under this background, we conducted a retrospective cohort analysis of RCC patients at the pT3 stage to detect whether they benefited from LND.

\section{Subjects/Patients and Methods}

\section{Clinical information}

In this study, 245 consecutive pT3 kidney cancer patients, who underwent open radical nephrectomy (RN) or laparoscopic RN with or without regional LND between January 2006 and December 2013 were included. pT3 was defined by the 2018 TNM system, which brought collective system invasion back into the standard. Lymph node invasion (LNI) was confirmed by two independent pathologists. All lymph node involvement/progressions were included.The exclusion criteria was failing to judge LN status (maybe ganglion), lack of clinical information and ectopic kidney tumor.

The clinical stage was based on the 2018 AJCC staging system. Among these patients, 63 (16.83\%) were classified as having LNI, and 311 patients were negative for LNI. Table 1 shows the clinical and pathological characteristics of the cohort. The study was undertaken with approval from the People's Liberation Army General Hospital (PLAGH) institutional ethics committee board. All of the patients signed the approval statement.

\section{Analytical methods}

The clinicopathologic features were summarized with means/medians and compared by whether an LND was conducted, using the Student's t-test, Wilcoxon test, and the chi-square test. The propensity score for whether an LND was conducted was obtained using a logistic regression model with LND as the outcome and the features in Table 1 as covariates. A total of 134 patients formed the cohort for the propensity score (PS) analysis, which included 67 patients that underwent LND and 67 patients that did not. Then we calculated the effect size for the paired data and visualize the distribution of PS result.

The association of LND with the development of progression-free survival (PFS), cancer-specific survival (CSS), and overall survival (OS) was evaluated with Cox models using several PS techniques and summarized hazard ratio (HRs) and 95\% confidence intervals (CIs). The PS techniques included 1:1 PS matching ( $\mathrm{n}=67$ pairs).

Then we selected pT3 RCC patients from TCGA database and extracted their overall survival time to conduct the K-M survival analysis as the external data set to test our conclusions.

At last, we divided patients into two groups based on the LN number. Then we further compared the survival time between the extended LND group (at least $5 \mathrm{LNs}$ ) and LND group (less than $5 \mathrm{LNs}$ ).

Statistical analyses were performed with the $\mathrm{R}$ software environment (version 3.4.0; http://r-project.org/), and $p$ values $<0.05$ were considered statistically significant.

\section{Results}

In total, 245 patients underwent $\mathrm{RN}$ for $\mathrm{pT} 3$ RCC, including 67 (27.3\%) with LND, and 21 (8.57\%) patients had LNI. The median number of LNs removed was 3 (1-18). The clinicopathologic features were compared using statistical methods, and we found that the tumor diameter and pathology results showed significant differences between the LND cohort and no LND cohort. Between the groups of with or without sarcoma, open surgery or laparoscopic surgery also showed a trend of differences. After balancing with the PS adjustment, none of the parameters showed a statistical difference. The median follow-up was 4.9 years. During this time, $80(59.7 \%)$ patients had tumor progression, 64 patients died from RCC, and 70 patients survival. The balanced data, after PS matching, is shown in a distribution figure (Figure 1).All of the effective sizes were less than 0.2 that means the balance was successful.

Then, we conducted a Kaplan-Meier (K-M) survival analysis with the data for the matched pairs. The associations of LND with PFS, CSS, and OS were shown in Figure 2, Figure 3, and Figure 4, respectively. Then, we developed a Cox analysis to detect whether LND can be a risk factor for PFS, CSS, and OS. The results showed that LND was not significantly associated with PFS, CSS, or OS. The HRs for PFS, CSS, and OS were 1.19 (95\% CI: 0.7624-1.856, $p=0.444), 1.064$ (95\% CI: 0.6439-1.758, $p=0.809)$, and 1.059 (95\% CI: 0.6557-1.709, $p=0.816$ ), respectively. As 
the M1 RCC patients were also included in the cohort, we then conducted another K-M survival analysis with the pT3M0 patients, and there was also no survival difference (Figure S1, S2, S3). We also analysed whether LND can benefit the RCC patients with inferior vena cava tumor thrombus which spread in the pT3a-pT3c and we got negative PFS and OS (Figure S4).

Within the TCGA database (2010-2014), we identified 182 pT3 renal cell carcinoma patients underwent RN with or without LND. Among them,
57 patients were M1, 121 were M0, and 4 were MX (deleted). Then we conducted K-M survival analysis in all patients and M0 patients for OS as their recurrence information is deficient. The results validated our previous conclusion that the survival time showed no difference between the LND group with the no LND group, HRs were $1.0376(95 \% \mathrm{Cl}$ : $0.9638-1.587, p=0.865)$ and $1.019(95 \% \mathrm{Cl}: 0.5659-1.836$, $p=0.949$ ) in all patients and M0 patients, respectively (Figure 5, Figure 6).

Table 1. The clinical and pathological characteristics of the cohort.

\begin{tabular}{|c|c|c|c|c|c|c|c|}
\hline \multirow[b]{2}{*}{ Feature } & \multicolumn{3}{|l|}{ overall cohort $(n=245)$} & \multicolumn{3}{|c|}{ matched cohort( $n=134)$} & \multirow[b]{2}{*}{$\begin{array}{l}\text { Effect } \\
\text { Size }\end{array}$} \\
\hline & without LND(n=178) & LND (n=67) & P Value & without LND & LND & P Value & \\
\hline gender & & & 0.34129 & & & 0.853 & 0.123 \\
\hline male & 133 & 46 & & 45 & 46 & & \\
\hline female & 45 & 21 & & 22 & 21 & & \\
\hline mean(median)age & $53.95(55.00)$ & 53.51 & 0.821 & $\begin{array}{l}54.7 \\
(56.0)\end{array}$ & $\begin{array}{l}53.51 \\
(56.00)\end{array}$ & 0.629 & 0.054 \\
\hline & & $(56.00)$ & & & & & \\
\hline $\begin{array}{l}\text { mean(median) BMI } \\
(\mathrm{n}=194)\end{array}$ & $25.11(24.87)$ & $\begin{array}{l}24.61 \\
(25.04)\end{array}$ & 0.2821 & $\begin{array}{l}24.36 \\
(24.18)\end{array}$ & $\begin{array}{l}24.56 \\
(25.01)\end{array}$ & 0.787 & 0.074 \\
\hline ECOG PS & & & 0.495 & & & 0.771 & 0.097 \\
\hline 1 & 154 & 50 & & 60 & 60 & & \\
\hline$>1$ & 24 & 7 & & 7 & 7 & & \\
\hline mean(median)diameter & $6.342(6.000)$ & $7.51(8.00)$ & $0.0404^{*}$ & $\begin{array}{l}6.893 \\
(7.000)\end{array}$ & $\begin{array}{l}7.51 \\
(8.00)\end{array}$ & 0.377 & 0.115 \\
\hline symptoms & & & 0.0917 & & & 0.856 & 0.076 \\
\hline no & 136 & 44 & & 43 & 44 & & \\
\hline yes & 42 & 23 & & 24 & 23 & & \\
\hline necrosis & & & 0.365 & & & 0.471 & 0.036 \\
\hline no & 130 & 45 & & 41 & 45 & & \\
\hline yes & 48 & 22 & & 26 & 22 & & \\
\hline sarcoma & & & 0.191 & & & 0.573 & 0.123 \\
\hline no & 166 & 59 & & 61 & 59 & & \\
\hline yes & 12 & 8 & & 6 & 8 & & \\
\hline WHO/ISUP(n=201/n=96) & & & 0.2188 & & & 0.476 & 0.135 \\
\hline $1-2$ & 88 & 17 & & 23 & 17 & & \\
\hline $3-4$ & 63 & 28 & & 28 & 28 & & \\
\hline thrombosis & & & 0.209 & & & 0.299 & 0.035 \\
\hline no & 119 & 39 & & 33 & 39 & & \\
\hline yes & 59 & 28 & & 34 & 28 & & \\
\hline collecting system & & & 0.545 & & & 0.723 & 0 \\
\hline no & 74 & 25 & & 27 & 25 & & \\
\hline yes & 104 & 42 & & 40 & 42 & & \\
\hline fat invasion & & & 0.166 & & & 0.723 & 0.099 \\
\hline no & 123 & 40 & & 42 & 40 & & \\
\hline yes & 55 & 27 & & 25 & 27 & & \\
\hline metastasis & & & 0.102 & & & 0.484 & 0 \\
\hline no & 156 & 57 & & 30 & 26 & & \\
\hline yes & 22 & 10 & & 37 & 41 & & \\
\hline pathology & & & $0.0198^{*}$ & & & 0.249 & 0.104 \\
\hline ccRCC & 159 & 51 & & 55 & 51 & & \\
\hline $\mathrm{pRCC}$ & 8 & 7 & & 7 & 7 & & \\
\hline chRCC & 3 & 1 & & 0 & 1 & & \\
\hline collecting duct RCC & 4 & 6 & & 4 & 6 & & \\
\hline other types & 4 & 2 & & 1 & 2 & & \\
\hline operation & & & 0.152 & & & 0.598 & 0.186 \\
\hline open RN & 126 & 41 & & 38 & 41 & & \\
\hline laparoscopic RN & 52 & 26 & & 29 & 26 & & \\
\hline
\end{tabular}




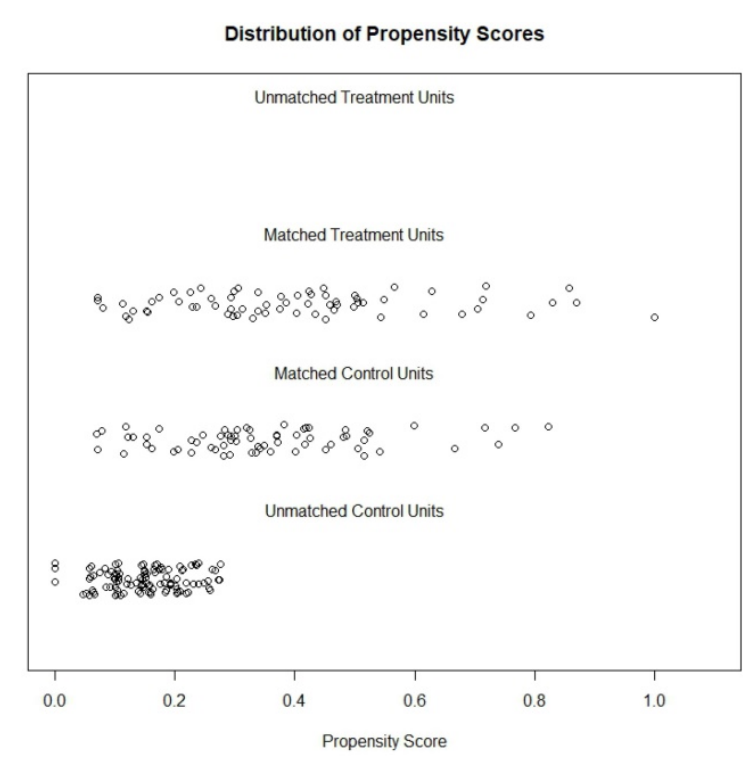

Figure 1. Distribution of the propensity scores.

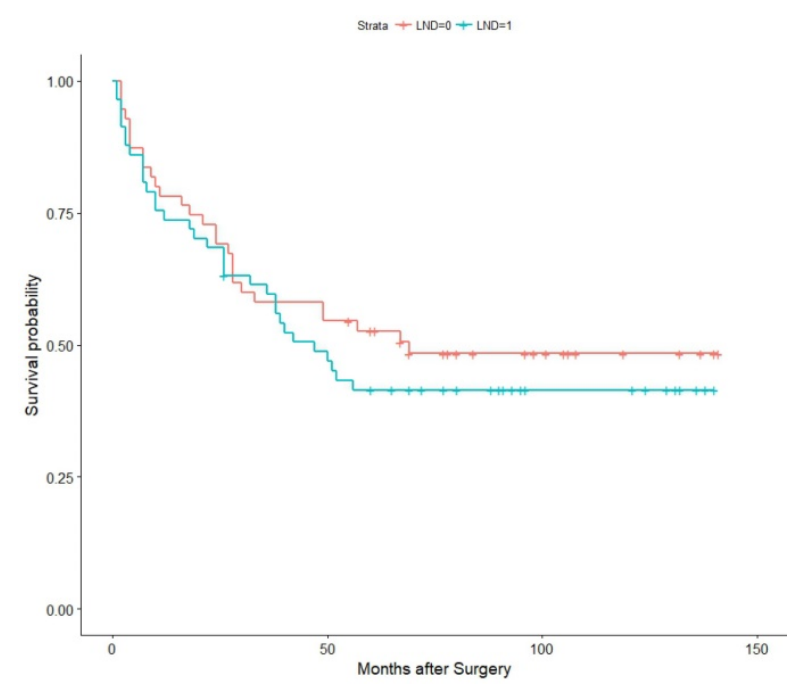

Figure 2. Association of LND with PFS among the subset of the 67 propensity score-matched pairs.

Considering the LND number may also influence the oncologic outcome, we defined the removal of at least $5 \mathrm{LNs}$ as extended LND and divided the patients into 2 groups: LND extended group (14 patients) and LND group (120 patients). The probability of LNI in LND extended group is $35.71 \%(5 / 14)$, but only $13.33 \%(16 / 120)$ in LND group. Interestingly, extended LND showed somehow negative effect on OS ( $\mathrm{HR}=1.8805,95 \% \mathrm{Cl}: 0.9299-3.803$, $p=0.0387$ ) in Figure 7, but CSS (HR=1.853,95\% CI: $0.9122-3.764, p=0.0832)$ and $\operatorname{PFS}(\mathrm{HR}=1.865,95 \%$ CI:0.9592-3.628, $p=0.0619$ ) failed to achieve statistical difference, just showing some tendency (Figure S6,S7).

\section{Discussion}

The role of LND in RCC surgery can be traced to
CJ Robson's research from 1969[9]. Regarding the issue of if LND can be beneficial for different subsets of RCC patients, the core problem became whether RCC patients will benefit or which subsets of patients will benefit. The only randomized evidence that the EORTC has provided failed to show a positive outcome, because they used the 1978 TNM system as a standard, which is quite different from today's standard, and the lymph node positive scale was so small that the conclusion was weakened.

During the past 2 decades, researchers have reduced the problem to whether any subset of RCC patients can benefit from LND. Given the fact that a benign lymph node dissection cannot improve survival and that the positive probability of T1 and T2 RCC is less than 4\% [10], several predictive models have been introduced with different clinicopathologic features to predict the probability of lymph node invasion; for the high probability $\mathrm{pN} 1$ patients, LND may play a more important role. However, noe of the models have been widely used in clinical practice.

Much prospective research has focused on T2-T4 RCC patients or metastatic RCC patients, and the researchers have drawn controversial conclusions. Herrlinger et al [11] reported that for T3a RCC patients, an extended lymphadenectomy provided the greatest benefits compared to T1 or T2 patients. On the other hand, Capitanio et al found that from the prognostic perspective, a staging lymphadenectomy appeared to be most valuable in patients with T1-2 RCC [12]. Schafhauser et al suggested that for T1-T4 patients, at least $4 \%$ of all patients benefit from an extensive lymphadenectomy [13]. However, many recent researches have drawn the opposite conclusion that they did not identify a benefit from LND in either non-metastatic or cytoreductive settings [2,14]. Gershman et al used propensity score techniques to balance the feather bias and reported that LND does not appear to provide a therapeutic benefit in patients with non-metastatic renal cell carcinoma [3]. Several researchers have focused on the question of whether mRCC patients can benefit from LND, and they usually reached a positive conclusion [6,15].

Even different systematic reviews regarding LND have conflicted each other. Capitanio et al reported in 2013 that an extended LND may be beneficial, when technically feasible, in patients with local T3-T4 RCC [16], but Bhindi et al did not find any positive conclusions and suggested that LND was not associated with improved oncological outcomes [17]. Another review remained neutral. Bekema et al indicated that there was no robust evidence to suggest superior oncologic outcomes or worse perioperative outcomes for RCC patients with LND at the time of 
nephrectomy, compared with patients who had a nephrectomy alone for CT3-T4 RCC [18].

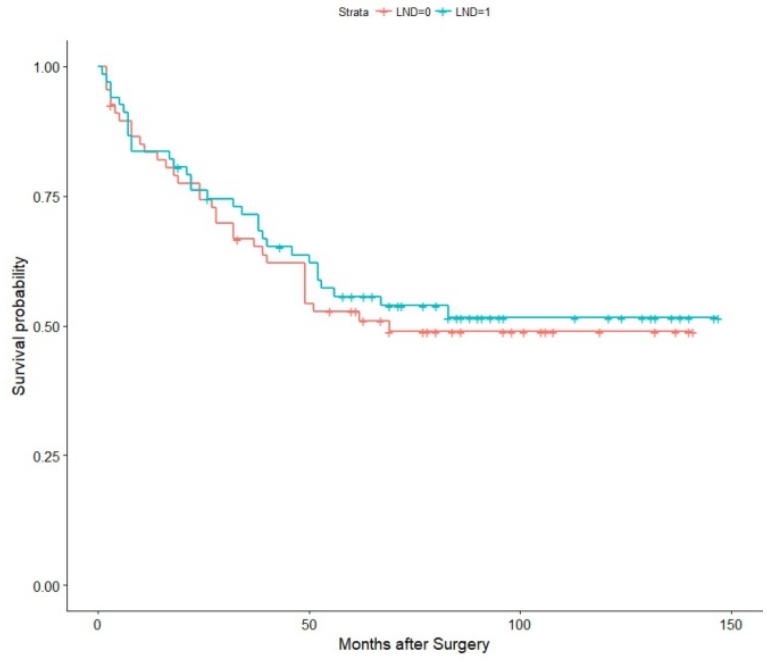

Figure 3. Association of LND with CSS among the subset of the 67 propensity score-matched pairs.

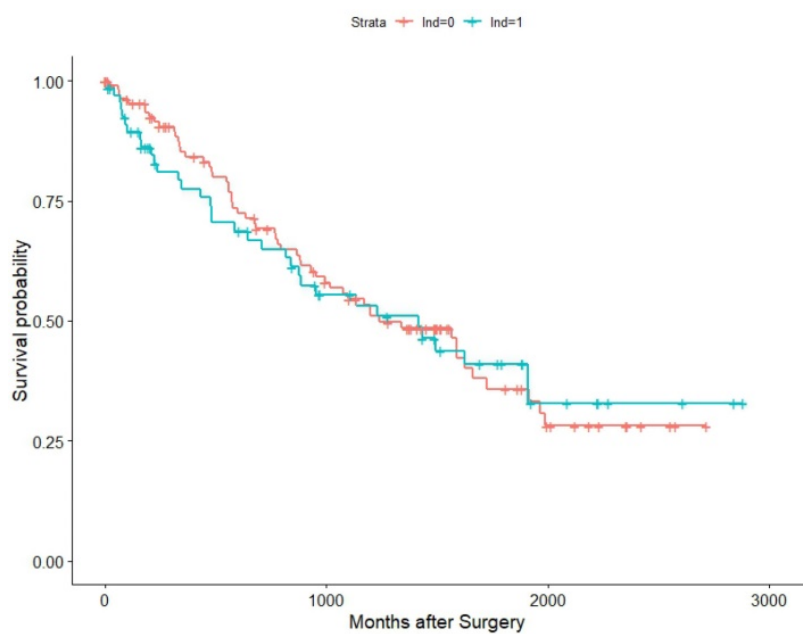

Figure 5. Association of LND with OS among the TCGA KIRC PT3 cohort.

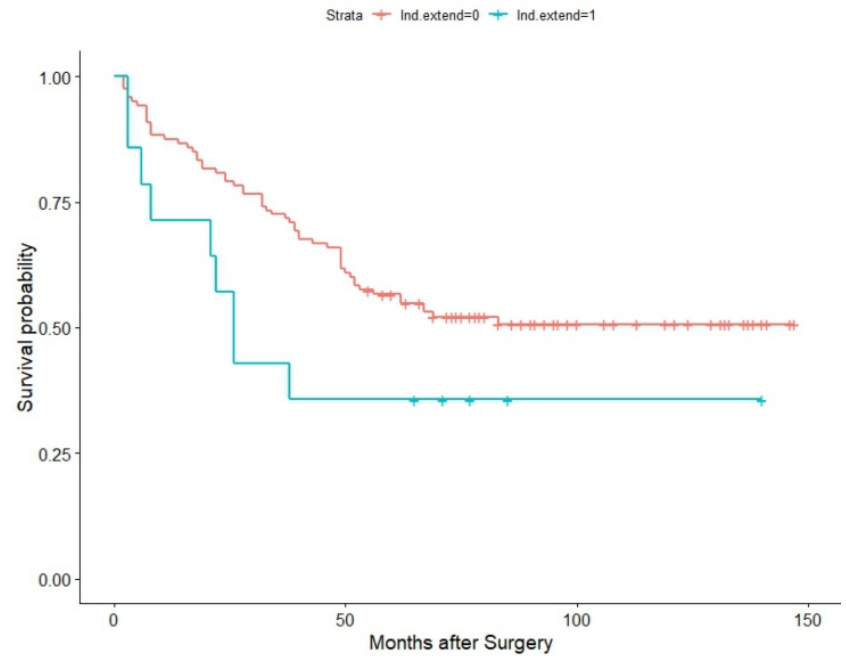

Figure 7. Association of LND with OS among the subset of the LND extend group (LND number $\geq 5)$ and the normal LND group $($ LND number $<5)$.

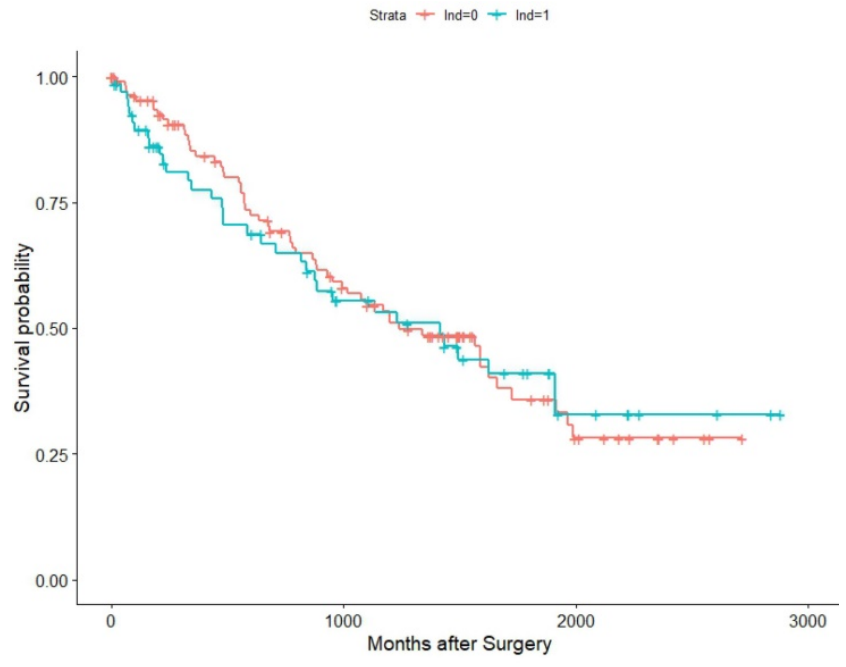

Figure 4. Association of LND with OS among the subset of the 67 propensity score-matched pairs.

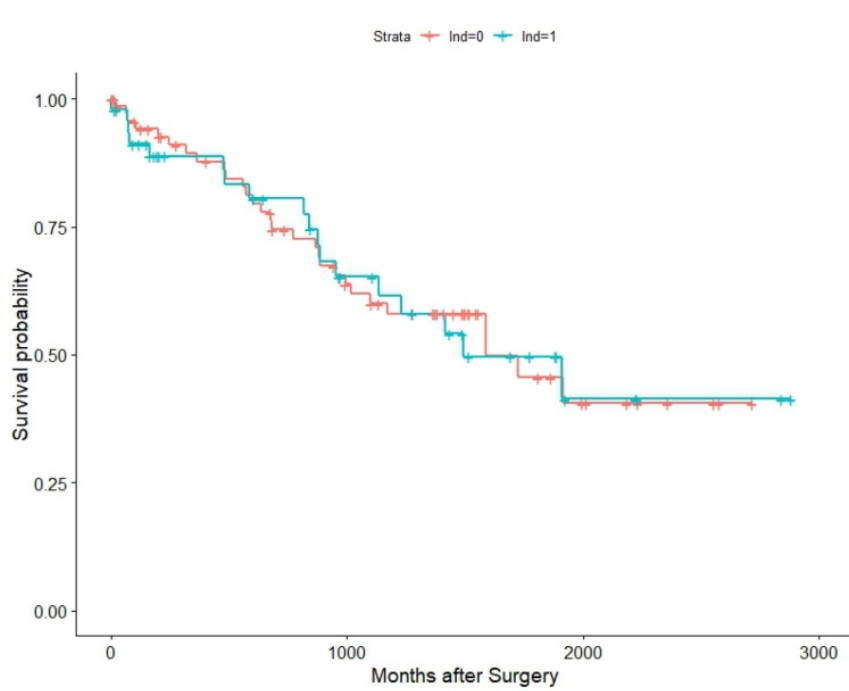

Figure 6. Association of LND with OS among the TCGA KIRC PT3M0 cohort.

The number of LND or the LND template is controversial too. Capitanio et al divided their patients into 3 groups that the mean numbers of nodes removed were 3.1, 9.7 and 14.8 in patients undergoing limited, regional and extended LND, respectively. They found that the LND number may affect the CSS in pT2 RCC patients[19], Marchioni et al defined the LND number as the number of removed nodes(NRN) and patients were split into two groups: $N R N \geq 3$ and NRN $<3$. Their finding showed that the NRN exerted a protective effect on CSS in pT2 but not in pT3 cohort[20]. Russo et al categorized their cohort into 3 groups based on the LND number $(0-3,4-7, \geq 8)$ and they did not find any significant difference between LND number and PFS and OS[15]. Leibovich et al set the LND number cut-off point as 13, and they also got negative results in PFS and OS. Our findings that the LND number at least 5 show a negative effect on OS was opposite to the previous study. This is 
probably because the extended LND group always has a larger tumor diameter (10 vs 6.875, $p=0.0004$ ). In the RCC surgery practice, the LND number varies a lot from country to country and surgeon to surgeon. This makes it difficult to standardize different study. As the various LND template exists, the LND number as a standard requires more discussion.

In our study, we have collected the largest single-center RCC patient dataset in China. With the background that the AJCC updated the TNM system standards, the invasion of the pelvicalyceal system joined the system again as a poor prognostic factor. Considering the fact that the controversy over LND has mostly focused on T3 and T4 RCC, and T3 stage is consisted of three heterogeneous factors, we attempted to examine a pT3 only retrospective cohort and utilized the PS score to calibrate our results.

Several limitations restricted our research. First, the standards of LND have not been defined. An extended LND and standard LND were regarded as a whole LND in our research. Furthermore, the standard regarding whether to perform an LND is also ambiguous. It mostly depends on the particular surgeon's experience. However, we matched open nephrectomy and laparoscopic nephrectomy as a propensity parameter to reduce the bias from different approaches. Several reports $[5,19]$ have pointed out that an extended LND may be more often recommended for pT3 RCC, at least to improve the PFS. These findings need future study. Second, the simple scale was limited, as LND is not prevalent in Chinese clinical practice, from the lack of definitive benefit but improving the anesthesia time and difficulty. Third, this research was limited by its retrospective and non-randomized design. To perform the propensity score analysis, 111 patients were excluded, which weakened the influence of the data. As the low probability of lymph node invasion and the nephron-sparing surgery become more and more popular, there is little chance for a prospective, randomized-controlled trial to be conducted worldwide, but multiple centers and larger samples cohort retrospective trials are also needed in the future. LND or extended LND may still be a method to be recommended for some subsets of RCC patients, but we do not know the classification methods.

\section{Conclusion}

Radical nephrectomy with LND cannot improve PFS, CSS, or OS for PT3 RCC patients.

\section{Supplementary Material}

Supplementary figures and tables. http://www.jcancer.org/v10p2369s1.pdf

\section{Acknowledgements}

This study was supported by the National Natural Science Foundation of China (Grant No. 81702492).

\section{Competing Interests}

The authors have declared that no competing interest exists.

\section{References}

[1] Capitanio U, Leibovich BC. The rationale and the role of lymph node dissection in renal cell carcinoma. World J Urol 2017;35:497-506. doi:10.1007/s00345-016-1886-3.

[2] Feuerstein MA, Kent M, Bazzi WM, et al. Analysis of lymph node dissection in patients with $\geq 7-\mathrm{cm}$ renal tumors. World J Urol. 2014;32:1531-6. doi:10.1007/s00345-013-1233-x.

[3] Gershman B, Thompson RH, Moreira DM, et al. Radical Nephrectomy With or Without Lymph Node Dissection for Nonmetastatic Renal Cell Carcinoma: A Propensity Score-based Analysis. Eur Urol. 2017;71:560-7. doi:10.1016/ j.eururo.2016.09.019.

[4] Bekema HJ, MacLennan S, Imamura M, et al. Systematic Review of Adrenalectomy and Lymph Node Dissection in Locally Advanced Renal Cell Carcinoma. Eur Urol. 2013;64:799-810. doi:10.1016/j.eururo.2013.04.033.

[5] Whitson JM, Harris CR, Reese AC, et al.. Lymphadenectomy Improves Survival of Patients With Renal Cell Carcinoma and Nodal Metastases. J Urol. 2011;185:1615-20. doi:10.1016/j.juro.2010.12.053.

[6] Pantuck AJ, Zisman A, Dorey F, et al. Renal Cell Carcinoma With Retroperitoneal Lymph Nodes: Role of Lymph Node Dissection. J Urol. 2003;169:2076-83. doi:10.1097/01.ju.0000066130.27119.1c.

[7] Crispen PL, Breau RH, Allmer C, et al. Lymph Node Dissection at the Time of Radical Nephrectomy for High-Risk Clear Cell Renal Cell Carcinoma: Indications and Recommendations for Surgical Templates. Eur Urol 2011;59:18-23. doi:10.1016/j.eururo.2010.08.042.

[8] [Internet] Ljungberg B (Chair), Albiges L, Bensalah K, et al. EAU Guildelines on Renal Cell Carcinoma 2018. https://uroweb.org/guideline/ renal-cell-carcinoma.

[9] Robson CJ, Churchill BM, Anderson W. The Results of Radical Nephrectomy for Renal Cell Carcinoma. J Urol. 1969;101:297-301. doi:10.1016/S0022-5347 (17)62331-0.

[10] Blom JHM, van Poppel H, Maréchal JM, et al. Radical Nephrectomy with and without Lymph-Node Dissection: Final Results of European Organization for Research and Treatment of Cancer (EORTC) Randomized Phase 3 Trial 30881. Eur Urol. 2009;55:28-34. doi:10.1016/j.eururo.2008.09.052.

[11] Herrlinger A, Schrott KM, Schott G, et al. What are the Benefits of Extended Dissection of the Regional Renal Lymph Nodes in the Therapy of Renal Cell Carcinoma? J Urol. 1991;146:1224-7. doi:https:/ / doi.org/10.1016/S0022-5347 (17)38052-7.

[12] Capitanio U, Jeldres C, Patard J-J, et al. Stage-specific effect of nodal metastases on survival in patients with non-metastatic renal cell carcinoma. BJU Int. 2009;103:33-7. doi:10.1111/j.1464-410X.2008.08014.x.

[13] Schafhauser W, Ebert A, Brod J, et al. Lymph node involvement in renal cell carcinoma and survival chance by systematic lymphadenectomy. Anticancer Res. 1999;19:1573-1578.

[14] Feuerstein MA, Kent M, Bernstein M, et al. Lymph node dissection during cytoreductive nephrectomy: A retrospective analysis: Node dissection in metastatic renal cell. Int J Urol. 2014;1:874-9. doi:10.1111/iju.12457..

[15] Lughezzani G, Capitanio U, Jeldres C, et al. Prognostic significance of lymph node invasion in patients with metastatic renal cell carcinoma: A population-based perspective. Cancer. 2009;115:5680-7. doi:10.1002/cncr. 24682.

[16] Capitanio U, Becker F, Blute ML, et al. Lymph Node Dissection in Renal Cell Carcinoma. Eur Urol. 2011;60:1212-20. doi:10.1016/j.eururo.2011.09.003.

[17] Brito J, Gershman B. The role of lymph node dissection in the contemporary management of renal cell carcinoma: A critical appraisal of the evidence. Urol Oncol Semin Orig Investig. 2017;35:623-6. doi:10.1016/j.urolonc.2017.06.054.

[18] Bekema HJ, MacLennan S, Imamura M, et al. Systematic Review of Adrenalectomy and Lymph Node Dissection in Locally Advanced Renal Cell Carcinoma. $\quad$ Eur Urol. 2013;64:799-810. doi:10.1016/j.eururo.2013.04.033. 
[19] Capitanio U, Suardi N, Matloob R, et al. Extent of lymph node dissection at nephrectomy affects cancer-specific survival and metastatic progression in specific sub-categories of patients with renal cell carcinoma (RCC): Effect of LND extent on RCC-specific survival and metastatic progression. BJU Int. 2014;114:210-5. doi:10.1111/bju.12508.

[20] Marchioni M, Bandini M, Pompe RS, et al. The impact of lymph node dissection and positive lymph nodes on cancer-specific mortality in contemporary pT 2-3 non-metastatic renal cell carcinoma treated with radical nephrectomy. BJU Int. 2018;121:383-92. 